Proceedings

\title{
High-Throughput Electrospinning of Bioactive Scaffolds for Bone Regeneration ${ }^{\dagger}$
}

\author{
Herman Novik ${ }^{1,2}, *$, Marta Clerici ${ }^{1}$, Amir Fahmi ${ }^{2}$, Matej Buzgo ${ }^{1}$ and Aiva Simaite ${ }^{1}$ \\ 1 InoCure s.r.o., R\&D Lab, Prumyslová 1960, 25088 Celákovice, Czech Republic; marta@inocure.cz (M.C.); \\ matej@inocure.cz (M.B.); aiva@inocure.cz (A.S.) \\ 2 Faculty of Technology and Bionics, Rhein-Waal University of Applied Sciences (HSRW), Marie-Curie 1, \\ 47533 Kleve, Germany; amir.fahmi@hsrw.org \\ * Correspondence: herman@inocure.cz \\ + Presented at the 1st International Electronic Conference on Pharmaceutics;St. Alban-Anlage 66, 4052 Basel, \\ Switzerland; 1-15 December 2020; Available online: https://iecp2020.sciforum.net/.
}

Citation: Novik, H.; Clerici, M.;

Fahmi, A.; Buzgo, M.; Simaite, A.

High-Throughput Electrospinning of

Bioactive Scaffolds for Bone Regen-

eration. Proceedings 2021, 78, 24.

https://doi.org/10.3390/

IECP2020-08666

Published: 1 December 2020

Publisher's Note: MDPI stays neutral with regard to jurisdictional claims in published maps and institutional affiliations.

Copyright: (ㄷ) 2020 by the authors. Licensee MDPI, Basel, Switzerland. This article is an open access article distributed under the terms and conditions of the Creative Commons Attribution (CC BY) license (http://creativecommons.org/licenses/by/4.0/).

\begin{abstract}
Among the most promising technologies for sustained drug delivery systems are coreshell nanofibres prepared by electrospinning. However, the most common method for the production of those, coaxial electrospinning, suffers from extremely low flow rates limiting the practical applications of such fibres. Emulsion electrospinning, on the other hand, enables the use of the highthroughput needle-less electrospinning devices for the production of the core-shell nanofibres with active pharmaceutical ingredients embedded and protected in their core. The development of such drug delivery systems for the applications in bone regeneration is further challenged by the need for the inorganic additives meant to stimulate the regeneration of the bone. The main objective of this work is to develop a high-throughput electrospinning method for the production of hybrid (organic-inorganic) and bioactive scaffolds needed for bone regeneration. We demonstrate the importance of the formulation, e.g., presence of surfactants, on the stability of the emulsion, and thus on electrospinning. Our work is an important step forward towards the high-throughput production of complex multi-material scaffolds for sustained drug delivery.
\end{abstract}

Keywords: high-throughput electrospinning; bioactive scaffolds; emulsion stability; emulsion electrospinning; core-shell nanofibres; drug release

\section{Introduction}

Osteoporosis is a bone disease that occurs due to a misbalance between the osteoblast and osteoclast functions [1]. This leads to the weakening of the bone structures and frequent bone fractures, that significantly affect the quality of life [2]. Regenerative medicine offers a potential to heal or replace the damaged tissues by providing therapeutics that combine cells, biomaterials and signalling molecules [3,4]. Hydroxyapatite (HAp), especially in nanosize, is one the most commonly used inorganic materials to promote bone regeneration [5]. However, hydroxyapatite alone is not enough to achieve full bone healing in elderly patients with limited regeneration capability. Our aim is to develop hybrid scaffolds that combine inorganic materials (HAp) with growth factors using the emulsion electrospinning. The main challenge addressed in this work is the preparation of stable emulsion for high-throughput applications.

Emulsion electrospinning is an emerging technology for the preparation of the coreshell fibres for drug delivery. Its main advantage is the possibility to upscale the production of such fibres using needleless electrospinning techniques [6]. However, one of the main remaining challenges of the technology is the stability of the emulsion - the solution needs to remain stable during electrospinning and under the applied electric field. In order to obtain stable emulsions, the surfactants and the composition of the oil and water 
phases have to be carefully balanced. In this work, we have tested multiple combinations of surfactants in order to enable the high-throughput electrospinning (at $30 \mathrm{~mL} / \mathrm{h}, 8.2 \mathrm{~g} / \mathrm{h}$ ). Specifically, we have tested the influence of three surfactants (P31R1, PVA, PF68) on emulsion stability (characterized visually) and fibre morphology. Release rates of encapsulated proteins were also tested but are not presented in this work. Finally, using the optimized formulation, we were able to combine the core-shell fibres with the inorganic additives that may enable the preparation of yet unreported hybrid nanofibres.

\section{Experiments}

Materials. Polymers and surfactants: $45 \mathrm{kDa}$ polycaprolactone (PCL) from Sigma Aldrich, 5-88 kDa PVA Emprov from Merck, Pluronic F-68 (PF68) with Mw 8400 Da from PanReac AppliChem, Pluronic-P31R1 with Mw 3300 Da from Sigma Aldrich. Solvents: chloroform (stabilized with Ethanol 0.6\%), ethanol absolute (EtOH, 99.7\% purity), water (technical) from VWR. Active ingredients: hydroxyapatite (HAp, $200 \mathrm{~nm}$ ) from Sigma Aldrich, albumin-fluorescein isothiocyanate conjugate (FITC-BSA, Mw $=10 \mathrm{kDa}$ ), tetramethylrhodamine isothiocyanate-Dextran (TRITC-dextran, $\mathrm{Mw}=40 \mathrm{kDa}$ ) from Sigma-Aldrich.

Stock solutions. In all experiments, stock solutions of polymers were prepared and diluted to needed concentrations prior to the experiment. The following solutions were used: $36 \%(w / v)$ PCL in chloroform, with $0.2 \%(v / v)$ of P31R1 in chloroform. As inorganic component, $30 \% w / v$ of HAp was added directly into the PCL stock and mixed. PVA $10 \%$, PF68 20\%, $10 \mathrm{mg} / \mathrm{mL}$ of BSA-FITC and TRITC-dextran were used for the water phase.

Preparation of emulsions. The general preparation sequence for $50 \mathrm{~mL}$ of the solution for the electrospinning was the following: $5 \mathrm{~mL}$ of water phase, comprising the mixture of PF68, PVA and protein or fluorescent dextrane conjugate in needed concentrations, and $10.5 \mathrm{~mL}$ of chloroform were mixed. When needed, P31R1 was added into the organic phase. The obtained combination was premixed by hand. Then, $5 \mathrm{~mL}$ of PCL stock solution was added and the mixtures were homogenized for $2 \mathrm{~min}$ at $6600 \mathrm{rpm}$ using the IKA device T-18 Digital ULTRA TURRAX. After homogenization another remaining amount of PCL stock with $5 \mathrm{~mL}$ of ethanol was added. As the last step manual mixing has been performed for approximately $2 \mathrm{~min}$. In all further discussions the percentage of the polymer mentioned refers to the percentage in each phase separately and not to the weight percent in fibres.

Evaluation of the stability of the emulsions. The changes to the emulsion appearance in time were observed, and the separation was quantified visually (semi-quantitatively). In all cases, the emulsions were separating into two distinct phases with water accumulating on the top. Based on the amount of the water observed the following levels demonstrated in Figure 1 were established: 1. Completely stable emulsion, no signs of separation; 2. First slightly distinguishable changes/water phase-related bubbles start to appear; 3 . Start of visible separation/big-sized bubbles; 4 . Circumferential separation, still with a slightly stable central part; 5 . Completely separated into two clearly distinguishable water and oil phase layers.

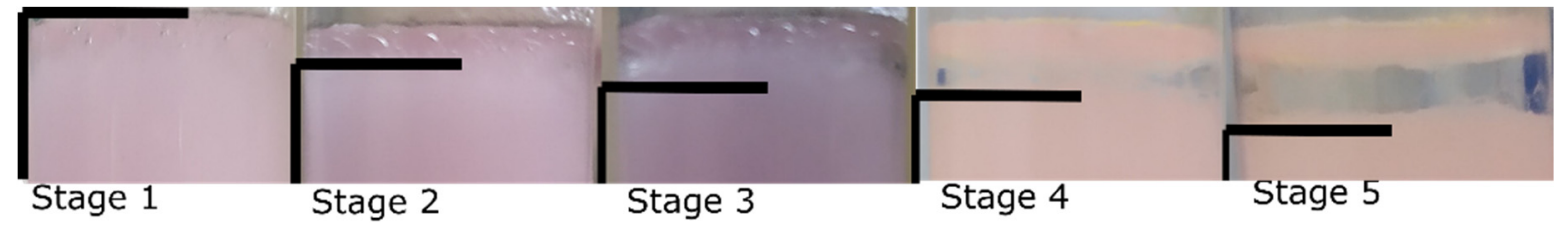

Figure 1. Visual representation of defined emulsion stability stages from completely stable 1 (on the left side) down to completely separated 5 (on the right side). 
Electrospinning. All electrospinning experiments were done using the lab-scale electrospinning unit from InoCure s.r.o. (InoSpin). For all experiments, a rotating drum collector with $10 \mathrm{~cm}$ diameter and a set speed of $500 \mathrm{rpm}$ was used, and the fibres were collected on the baking paper. A metallic cylinder (G10 needle) was used as a spinneret. The distance between the spinneret and the collector was $20 \mathrm{~cm}$. The electrospinning parameters used in all experiments were the following: Applied voltages were +55 and $-25 \mathrm{kV}$ with a flow rate range of $30-60 \mathrm{~mL} / \mathrm{h}$. The temperature and humidity inside the chamber were controlled by the integrated climate control unit InoCool (from InoCure s.r.o. Celákovice, Czech Republic) and were set to $10^{\circ} \mathrm{C}$ and $60 \%$, respectively.

Fibre characterization. Fibre morphology was characterized using the desktop scanning electron microscope (SEM) from Phenom-World. Before imaging, samples were sputter-coated using the SC7620 Mini Sputter Coater/Glow Discharge System from Quorum Technologies. Fibres were also characterized using the confocal microscopy on Zeiss LSM 880 Airyscan at the Institute of Experimental Medicine (IEM, Prague, Czech Republic). The following wavelength settings were used for the fluorophores: FITCBSA $-488 \mathrm{~nm}$ and TRITC-Dextran-561 nm.

\section{Results}

In this work, the factors affecting the stability of the emulsion were investigated. In agreement with previous works, we have determined that the high-speed homogenization is needed in order to improve the stability of the emulsion (data not shown) [7]. Thus, for all experiments, high-speed homogenization was used. Moreover, our preliminary data suggested that the water phase should be kept low in comparison to the oil phase, and for all experiments discussed here the oil to water ratio was fixed to 9:1. Finally, we hypothesized that in order to stabilize the emulsion, the increase in viscosity and density of the water phase is needed. The influence of PVA was tested in combination with other surfactants: PF68 and P31R1. The results on emulsion stability and fibre morphology were compared for fibres with and without P31R1, PF68 concentrations varying from 0 to $10 \%$ and PVA concentrations varying from 0 to $5 \%$. Finally, all the electrospinning experiments were done using high-throughput electrospinning with a $30 \mathrm{~mL} / \mathrm{hr}$ flow rate. The obtained results are summarized in the following sections.

\subsection{Emulsion Stability}

The influence of the combination of the surfactant on the stability of the emulsions is summarized in Figure 2. In all cases, the addition of the PF68 or PVA to the water phase significantly enhanced the stability of the emulsion. That is, with an increasing amount of PF68, the complete separation time was shifted from $20 \mathrm{~min}$ (in case of no PVA) to $30 \mathrm{~min}$ for the otherwise similar compositions. The increase in the amount of PVA had the same effect: the stability of the emulsion with, e.g., 5\% PF68 increased from $20 \mathrm{~min}$ for complete separation to 90 min once 5\% PVA was added. As shown in Figure 2, the same trend was observed in cases with and without P31R1 in the oil phase. In all cases, when none, or only one of the polymers was used (black lines in the figures), the emulsion separated in less than half an hour. However, the stability of the emulsion notably increased when both polymers were used together. The presence of P31R1 seems to improve the stability of the emulsion, but only in the presence of other surfactants (PF68 and PVA). 


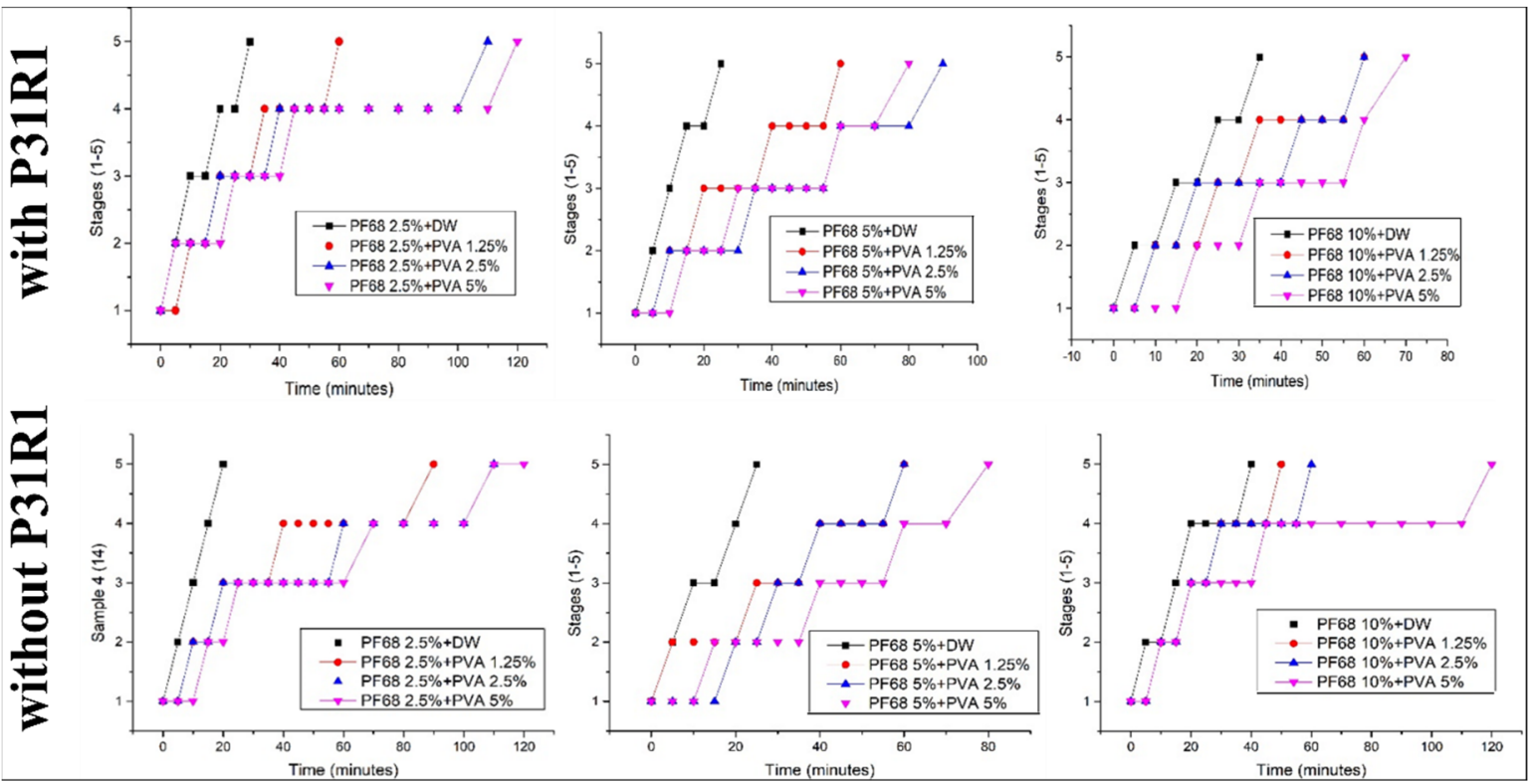

Figure 2. Stability recordings comparing samples with and without P31R1 surfactant in three different concentrations of PF68 (2.5, 5, 10\%) with the respective concentrations of PVA $(0,1.25,2.5,5 \%)$.

\subsection{Emulsion Electrospinning}

We have observed in our preliminary experiments that the electrospinning process is influenced by the stability of the emulsions. It was noted that if the solution separates, large defects are observed on the nanofibre mesh. In all cases, similar nanofibres with a size of $500 \mathrm{~nm}$ were obtained (not shown). In the SEM images shown in Figure 3, low magnification pictures were used, to visualize the feasibility of the electrospinning process.

The best electrospinning results were obtained with P31R1 and low amounts of PF68. In this case, for all PVA concentrations, nearly no large defects were detected. Moreover, some improvements in fibre morphology were detected with increasing PVA concentration. Interestingly, higher amounts of PF68 seem to increase the number of defects (compare $2.5 \%$ of PF68 and 10\% of PF68 in both figures). Finally, once P31R1 was used, only formulations with low PF68 showed fibres with few defects, whereas when no P31R1 was added, a high PF68 concentration (10\%) led to defectless fibre formation. These results suggest that PF68 and P31R1 may be playing a complimentary role in emulsion stabilization. That is, an excess in the combination of the two may destabilize the process. More experiments are needed to verify this observation. Finally, there was a significant decrease in the number of defects in the formulation with PVA compared to the ones without (see $0 \%$ and $1.25 \%$ ). On the other hand, increasing the amount of PVA does not seem to further improve the electrospinning process.

The core-shell structure of the fibres was visualized using the confocal microscopy. Note that two fluorescently labelled molecules were added to the water phase of the emulsion to quantify the release: TRITC-Dextran (red) and FITC-BSA (green) (release data not shown in this work). No dye was added to the shell of the fibres. The SEM images and confocal microscopy images of the fibre made with only PF68, with only PVA and with both surfactants are shown in Figure 4. In the fibres made with no PVA, separated instances of fluorophores are seen. However, in the case of PVA only, a continuous core structure can be seen from the traces of both fluorescent dyes and the fluorophores that are homogeneously dispersed throughout the fibre mesh. However, even in this case, the two colours of the two dyes mostly appear separately, indicating a potentially insufficient mixing of the water phase. On the other hand, when both surfactants are used (10\% PF68 and 5\% PVA), a continuous core is observed. However, in this case, only red fluorescence was measured, with only few separated dots of the green colour. The reason behind the 
loss of one fluorophore in the case of the two surfactants used will be investigated in future experiments.

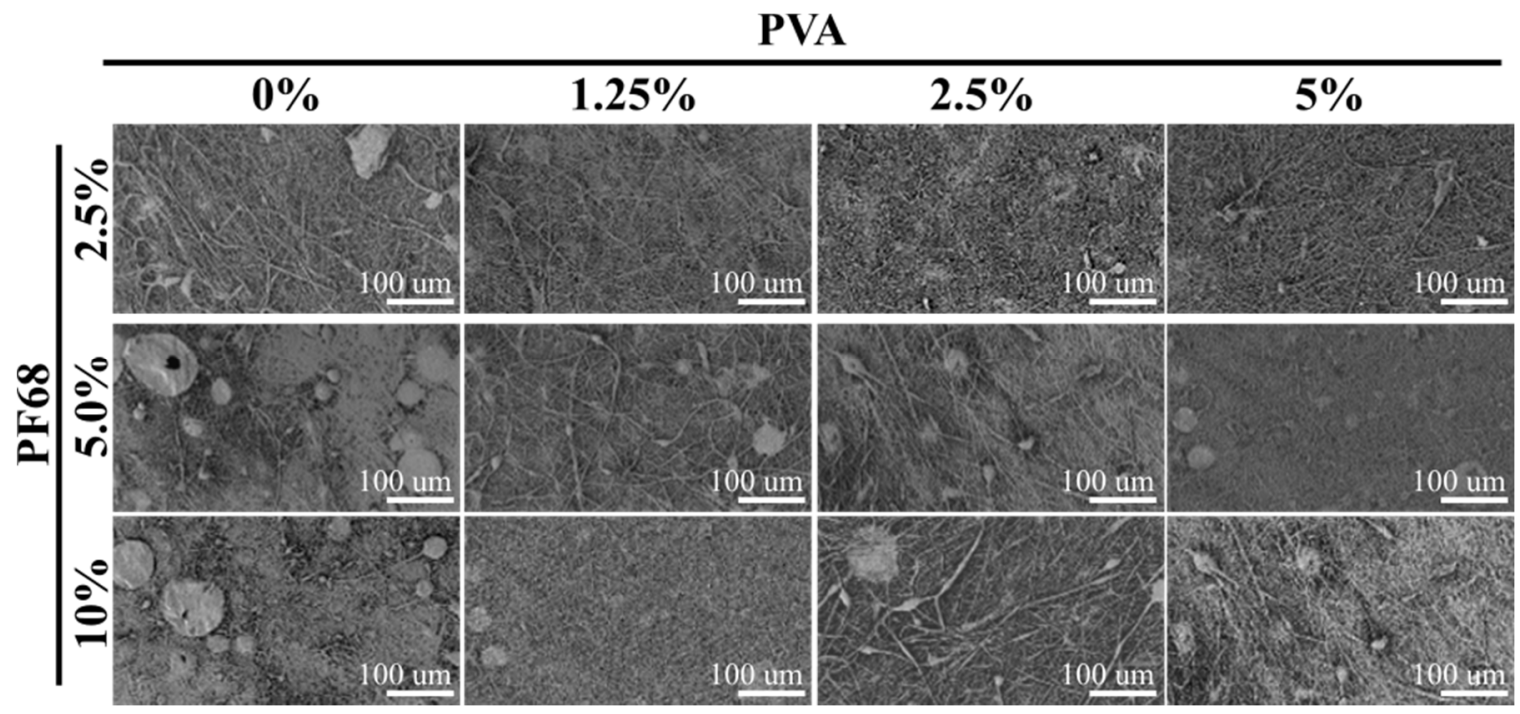

(a)

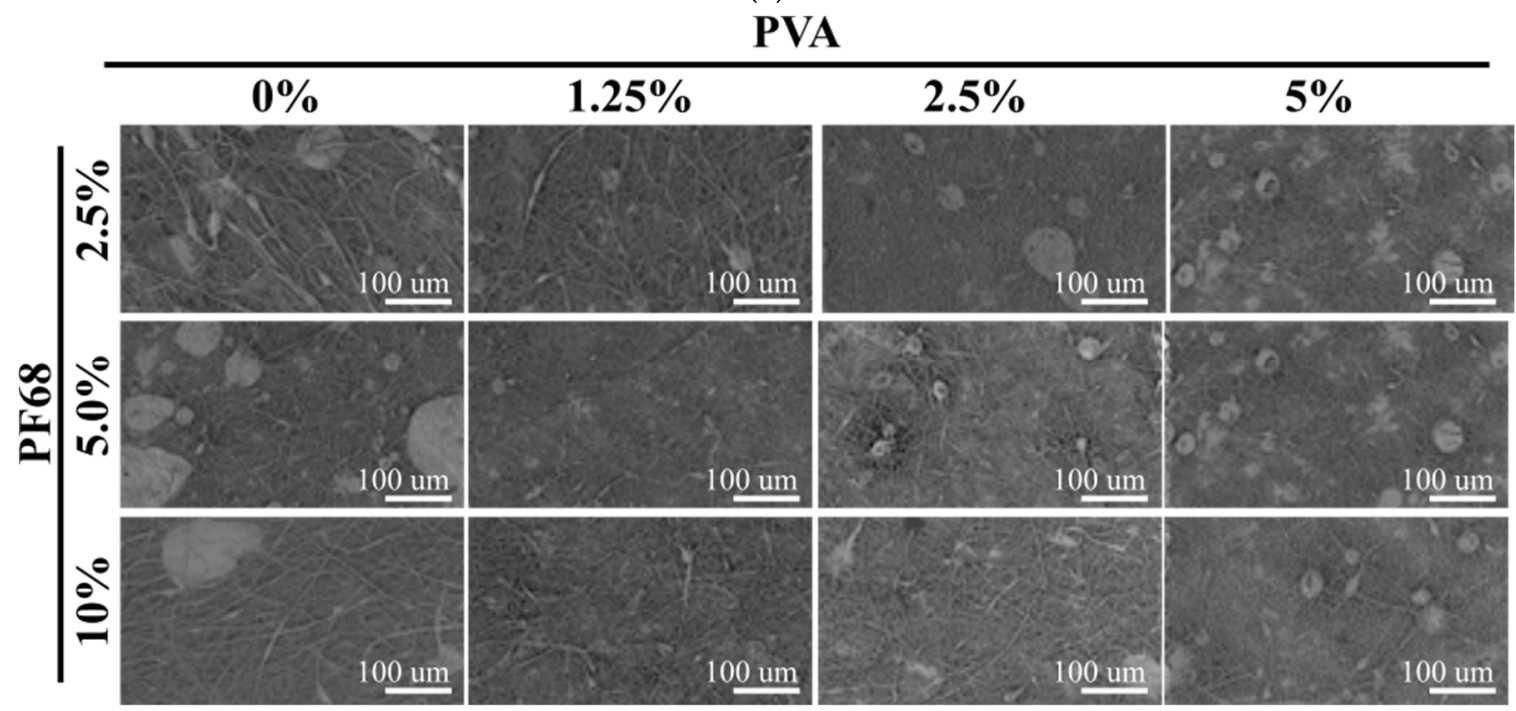

(b)

Figure 3. (a) SEM images of four different concentrations of PVA $(0,1.25,2.5,5 \%)$ in combinations with PF68 in three concentrations $(2.5,5,10 \%)$ with P31R1; (b) SEM images of four different concentrations of PVA $(0,1.25,2.5,5 \%)$ in combinations with PF68 in three concentrations $(2.5,5,10 \%)$ without P31R1.

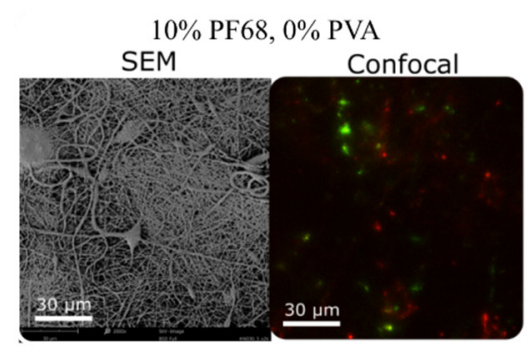

(a)

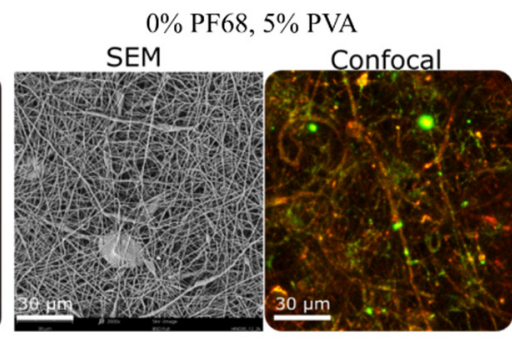

(b)

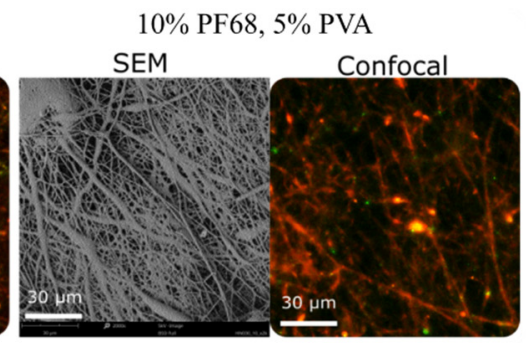

(c)

Figure 4. Comparison of SEM and confocal images between samples with (a) PF68 or (b) PVA only, as well as (c) mixture between PF68 and PVA. 


\subsection{Emulsion Electrospinning of HAp-Loaded Core-Shell Nanofibres}

Finally, scaffolds for bone regeneration require the release of not only the organic bioactive molecules, but also inorganic materials. To demonstrate the feasibility of the multimaterial scaffolds, core-shell nanofibres were prepared with the HAp in the PCL shell. In this case, emulsion with water and PF68 was used and as a reference, and fibres with no surfactants were made. The fibre morphology of the prepared scaffolds is shown in Figure 5. In all cases, mixed nano and micro fibres were made. Fibres made with HAp appear to be rougher, and clusters of HAp can also be observed, showing that HAp was successfully incorporated. Moreover, the improvement in morphology is clearly visible in Figure 5 from (a) to (c), where the best morphology was obtained with incorporated HAp. Interestingly, in this case, PVA was not needed in order to obtain fibres with few defects. This suggests that the presence of HAp may work as an additional emulsion stabilizer. Further work will be done to evaluate the influence of HAp on the emulsion stability and spinnability.

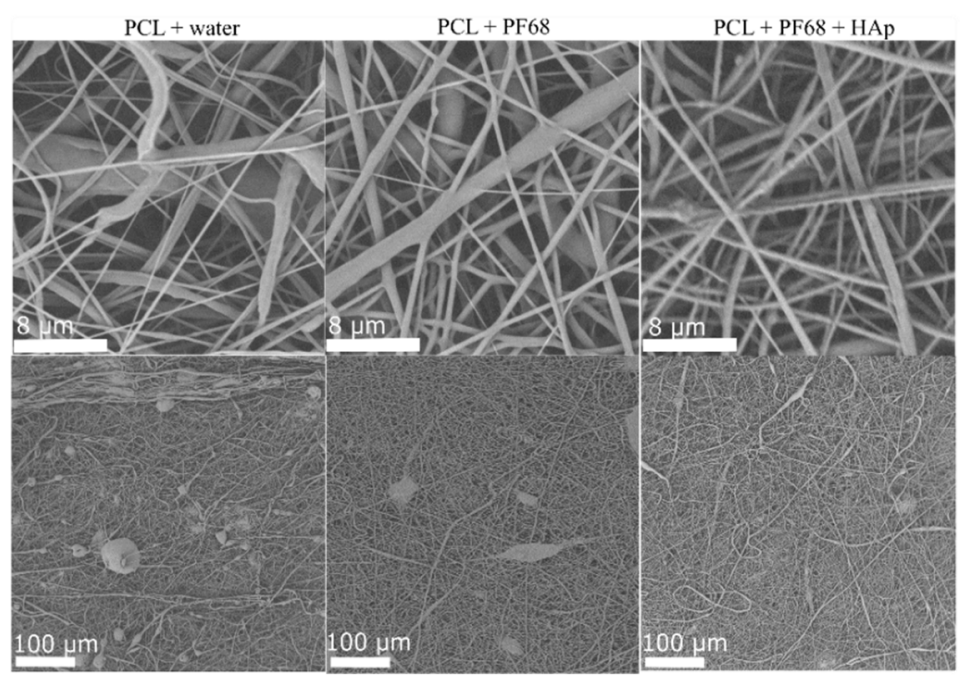

(a)

(b)

(c)

Figure 5. SEM images of sample (a) PCL + water, (b) PCL + PF68, (c) PCL + PF68 + HAp.

\section{Discussion}

Our results indicate that a careful selection of surfactants is needed in order to enable the high-throughput electrospinning of emulsions. We have demonstrated complex interactions between different surfactants and their influence on both emulsion stability and fibre morphology. We hypothesized that more stable emulsions would lead to better electrospinning results. However, the comparison of Figure 2 (stability quantification) and Figure 3 (fibre morphology) does not support this hypothesis. Our stability analysis suggested that increasing the PVA concentration increases the stability of the emulsion. However, an increase in the quality of the obtained electrospun scaffolds was only visible between the scaffolds with and without PVA. PVA concentration did not affect the quality and, in the case of the scaffolds without P31R1, may even lead to more frequent defects. The semi-quantitative observation of the emulsion stability did not correlate with the observation of better fibre mesh quality when PF68 was used without P31R1. Note that in all cases, only $15 \mathrm{~mL}$ of the solution were electrospun for the experiment. At a rate of 30 $\mathrm{mL} / \mathrm{hr}$, it took about $30 \mathrm{~min}$ to complete the process. Most of the formulations showed sufficient stability in $30 \mathrm{~min}$. Only the formulation without PVA and 10\% PF68 without P31R1 reached a level 4 separation in $30 \mathrm{~min}$. While further experiments are needed to verify this, it is likely that the emulsion stability is only needed for the time of electrospinning. In most of the cases where PVA was used, the stability was sufficient. 
The reasons behind the continuous and non-continuous core morphology and nonhomogeneous protein distribution in fibres are unclear as well. It was previously reported that proteins such as BSA would move to the surface of the nanofibres during the electrospinning if the $\mathrm{pH}$ of the water was not adjusted to proteins $\mathrm{pI}$ [8]. As shown in Figure 4, less of a green dye (BSA-TRITC) can be seen in the core-shell structure of the fibres with PF68. This suggests that water-soluble PF68 may facilitate such migration. The results also suggest that the addition of more viscous and linear PVA to the water phase may lead to the continuous core formation and homogeneous distribution of the active ingredients in the nanofibres. Both observations will be further investigated.

Finally, we have also demonstrated that emulsion electrospinning can also be used to produce more complex multi-material systems. In this case, a still non-optimal combination of the surfactants was used to make an organic-inorganic PCL shell in the coreshell nanofibres. Nevertheless, defectless and smooth fibres with HAp distributed throughout them (Figure 5c) were obtained. Further optimization of such complex fibres is needed. However, our results suggest high-throughput emulsion electrospinning can be used to produce the complex bioactive materials needed for tissue engineering applications.

\section{Conclusions}

We have investigated the influence of three surfactants (PF68, P31R1 and PVA) and their combination on the stability of the emulsions made for high-throughput electrospinning. We have shown that the addition of the PVA to the water phase notably increased the stability of the emulsion and the quality of electrospinning-less defects were produced with PVA in the water phase. However, further improvements in emulsion stability (e.g., by increasing the amount of PVA) did not seem to improve the electrospinning process. We suggest that it is important to make sure that emulsions are stable for the duration of the electrospinning, but overall emulsion stability is not correlated with quality. We have also investigated the influence of the surfactant on the core-shell structure and showed that PVA is needed in order to achieve a continuous core. Finally, emulsion electrospinning with inorganic additives in the shell was demonstrated. Overall, our work demonstrates various possibilities of high-throughput electrospinning for encapsulation of proteins in nanofibres.

Author Contributions: M.B., A.S. and H.N. conceived and designed the experiments; H.N. performed the experiments; H.N., M.B. and M.C. analysed the data; A.F. provided scientific guidance; M.B. contributed reagents/materials/analysis tools with subsequent discussions; H.N. and A.S. wrote the paper. All authors have read and agreed to the published version of the manuscript.

Funding: This research received no external funding.

Institutional Review Board Statement: Not applicable.

Informed Consent Statement: Not applicable.

Data Availability Statement: Not applicable.

Acknowledgements: This work was supported by the European Union's Horizon 2020 research and innovation programme under the Marie Skłodowska-Curie grant agreements no. 823981 (actiTOX) and no. 824007 (iP-Osteo). Novik, H. has received support from the Erasmus+ traineeship programme for the research activities at InoCure s.r.o.

Conflicts of Interest: The authors declare no conflict of interest. 


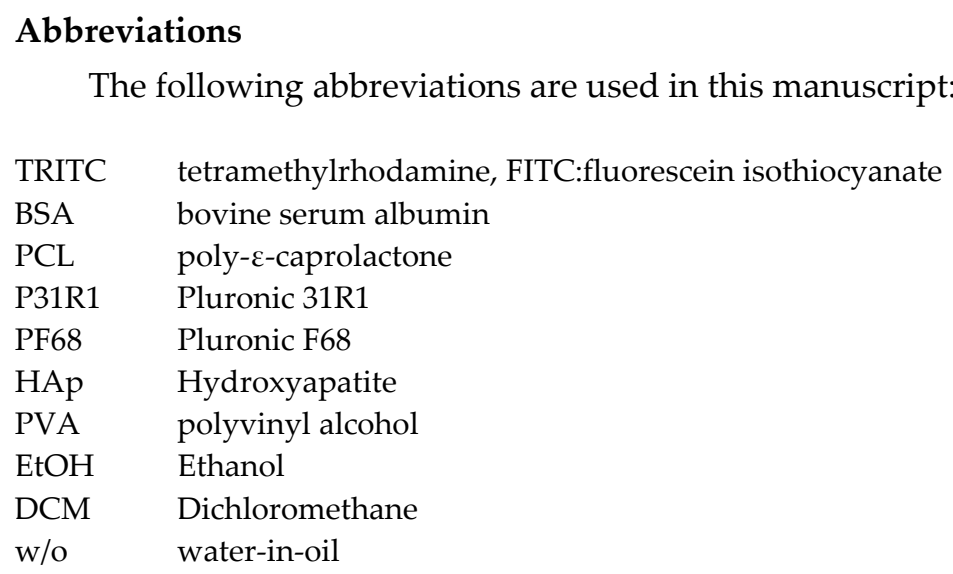

\section{References}

1. Akkawi, I.; Zmerly, H. Osteoporosis: Current concepts. Joints 2018, doi:10.1055/s-0038-1660790.

2. Goode, S.C.; Wright, T.F.; Lynch, C. Osteoporosis Screening and Treatment: A Collaborative Approach. J. Nurse Pract. 2020, doi:10.1016/j.nurpra.2019.10.017.

3. Sampogna, G.; Guraya, S.Y.; Forgione, A. Regenerative medicine: Historical roots and potential strategies in modern medicine. J. Microsc. Ultrastruct. 2015, doi:10.1016/j.jmau.2015.05.002.

4. Braghirolli, D.I.; Steffens, D.; Pranke, P. Electrospinning for regenerative medicine: A review of the main topics. Drug Discov. Today 2014, 19, 743-753, doi:10.1016/j.drudis.2014.03.024.

5. Bal, Z.; Kaito, T.; Korkusuz, F.; Yoshikawa, H. Bone regeneration with hydroxyapatite-based biomaterials. Emergent Mater. 2020, doi:10.1007/s42247-019-00063-3.

6. Buzgo, M.; Filova, E.; Staffa, A.M.; Rampichova, M.; Doupnik, M.; Vocetkova, K.; Lukasova, V.; Kolcun, R.; Lukas, D.; Necas, A.; et al. Needleless emulsion electrospinning for the regulated delivery of susceptible proteins. J. Tissue Eng. Regen. Med. 2018, doi:10.1002/term.2474.

7. Juttulapa, M.; Piriyaprasarth, S.; Takeuchi, H.; Sriamornsak, P. Effect of high-pressure homogenization on stability of emulsions containing zein and pectin. Asian J. Pharm. Sci. 2017, doi:10.1016/j.ajps.2016.09.004.

8. Tang, C.; Ozcam, A.E.; Stout, B.; Khan, S.A. Effect of pH on protein distribution in electrospun PVA/BSA composite nanofibers. Biomacromolecules 2012, doi:10.1021/bm2017146. 